\title{
EMPIRICAL ANALYSIS OF PERSISTENCE AND DEPENDENCE PATTERNS AMONG THE CAPITAL MARKETS $^{1}$
}

\author{
Miloslav Vošvrda*
}

\begin{abstract}
:
This paper investigates dependence structures on selected world stock markets. Firstly, a non-parametric univariate measure of a persistence concerning capital markets efficiency is derived and computed. Secondly, we focus on computing of a non-parametric multivariate measure of the persistence indicating an ability of the price mechanisms to hold capital market efficiency under interaction of shocks.
\end{abstract}

Keywords: dependence structure, non-parametric univariate - multivariate measures of the shock persistence, shock transmission

JEL Classification: C3, C13, G14

\section{Introduction}

An empirical analysis of dependence patterns in capital markets is based on both univariate and multivariate setting of the financial time series. Firstly, the efficient market hypothesis by Fama's definition (see Fama, 1970) in capital markets is analyzed. It means to identify a pure random walk property in the stock price trajectories and to analyze a capital market shock persistence ability. Such property is a typical property for a pure random walk. Secondly, we analyze a capital market transfer capability to launch financial shocks. At the same time, we analyze a propagation of these shocks among the others capital markets. In general, there are two categories of the non-pure random walk time series. The first category obtains the time series that can be viewed as a combination of a pure random walk process and a stationary process with non-zero serial correlation. The behaviour of these time series associated with their correlation structure is analyzed by the following two components: a trend which is a pure random walk, and a cycle which is a stationary process involving a serial correlation function. The shock persistence of the time

\footnotetext{
*) Institute of Information Theory and Automation, Academy of Sciences of the Czech Republic; Institute of Economic Studies, Faculty of Social Sciences, Charles University, Prague (vosvrda@vtia.cas.cz). This research was supported by the Ministry of Education of the Czech Republic under Project No. MSM0021620841, by the Grant Agency of the Czech Republic under Grant No. 402/05/0115, and the Grant Agency of Charles University under Grant No. 454/2004/A - EK/FSV.
} 
series depends on the relative contribution of these two components. Such time series mark the long-run effect smaller than that of a pure random walk. The second category obtains the time series that contain a unit root with its non-stationary component. If there is no stationary component in the time series then the first difference of the time series is a stationary process, and the long-run effect would be larger than that of a pure random walk. The shock persistence for selected capital markets will be estimated. Measures of the shock persistence in the time series in both univariate and multivariate case will be introduced.

Usually, the economic time series are formed by a combination of a non-stationary trend component, a stationary cycle component, and a noise component. Shocks to these components are different. They have remarkably different effects on future trend values. A shock to the stationary time series is transitory and the effect will disappear after a sufficiently long time. Let us consider the following simple first-order autoregressive process $\left\{y_{1, t}\right\}$ :

$$
y_{1, t}=c+\theta y_{1, t-1}+\varepsilon_{1, t}
$$

where $|\theta|<1,\left\{\varepsilon_{1, t}\right\}$ is an independent process of residuals with mean zero, and $c$ is a given constant. Suppose that there is a shock at time $t$ with its magnitude being $s$, and there is no shock afterwards, i.e.

$$
y_{1, t}=c+\theta y_{1, t-1}+\varepsilon_{1, t}+s .
$$

Then, after $k$ periods, the time series (2) has the following form:

$$
y_{1, t+k}=\frac{1-\theta^{k+1}}{1-\theta} c+\theta^{k+1} y_{1, t-1}+\theta^{k} s+\left(1+\theta L+\ldots+\theta^{k} L^{k}\right) \varepsilon_{1, t+k}
$$

where $L$ is the lag operator. Then the mean value is equal to

$$
E\left[y_{1, t+k}\right]=\frac{c}{1-\theta}+\theta^{k} s
$$

and

$$
\lim _{k \rightarrow \infty} E\left[y_{1, t+k}\right]=\lim _{k \rightarrow \infty} E\left(\frac{c}{1-\theta}+\theta^{k} s\right)=\frac{c}{1-\theta}
$$

It means that the time series revert to its mean value and the impact of the shock disappears; the smaller the value of the parameter $\theta$, the faster the impact disappears.

In contrast, a shock to a trend, as expressed in a pure random walk, moves the time series away from its trend path permanently by an extent which is exactly the size of the shock. Let us again consider the simple first-order autoregressive process $\left\{y_{2, t}\right\}$ but with $\theta=1$ :

$$
y_{2, t}=c+y_{2, t-1}+\varepsilon_{2, t}
$$

Assume that there is a shock at time $t$ with a magnitude of $s$, and there is no further shock afterwards. Then, after $k$ periods, the impact is to shift permanently the level of the time series by an extent of $s$ as follows:

$$
y_{2, t+k}=(k+1) c+y_{2, t-1}+s+\sum_{j=0}^{k} \varepsilon_{2, t+j}
$$


The impact will not disappear even if $k \rightarrow \infty$.

Now we can construct a convex combination of both a stationary time series of the type of equation (1) and a pure random walk such as equation (6), i.e., that $\alpha \in(0,1)$. After providing this convex combination we obtain the following simple first-order autoregressive process $\left\{y_{3, t}\right\}$ :

$$
y_{3, \mathrm{t}}=c+(1-\alpha(1-\theta)) \mathrm{y}_{3, \mathrm{t}-1}+\varepsilon_{3, t}
$$

where $\varepsilon_{3, t}=\alpha\left(\varepsilon_{1, t}-(1-\alpha)(1-\theta) \varepsilon_{1, t-1}\right)+(1-\alpha)\left(\varepsilon_{2, t}+\alpha(1-\theta) \varepsilon_{2, t-1}\right)$. If $\theta \rightarrow 1$, then the time series (8) is I(1) process. If $\theta \rightarrow 0$, then the time series (8) is I(0) process. We assume there is a shock at time $t$ with a magnitude of $s$, and there is no further shock afterwards. Then, after k periods, the impact of the shock will not disappear, nor the impact would be $s$ as follows:

$$
\begin{aligned}
y_{3, t+k}= & c \sum_{j=0}^{k}(1-\alpha(1-\theta))^{j}+(1-\alpha(1-\theta))^{k} y_{3, t-1}+ \\
& +(1-\alpha(1-\theta))^{k} s+\sum_{j=0}^{k}(1-\alpha(1-\theta))^{k-j} \varepsilon_{3, t+j}
\end{aligned}
$$

and

$$
\lim _{k \rightarrow \infty} E\left[y_{3, t+k}\right]=\lim _{k \rightarrow \infty}\left(\begin{array}{c}
\frac{c}{\alpha(1-\theta)}\left(1-(1-\alpha(1-\theta))^{k+1}\right)+ \\
+\left(1-(1-\alpha(1-\theta))^{k}\right)+ \\
+(1-\alpha(1-\theta))^{k} s
\end{array}\right)=\frac{c}{\alpha(1-\theta)} .
$$

It means that the convex combination of both the stationary time series of the type of equation (1) and the pure random walk such as equation (6) reverts to its mean value if $\alpha \rightarrow 1$ and the impact of the shock disappears; the smaller the value of the parameter $\theta$, the faster the impact disappears. Contrariwise, if $\alpha \rightarrow 0$ then the impact will not disappear.

Let us return to equation (2). The permanent impact of the shock would usually be smaller than $s$, depending on the relative contributions of the trend component and the cycle component. Furthermore, if $\theta \rightarrow 1$ in equation (1), then the first difference of the time series is $I(0)$ process and the impact of the shock will disappear after a sufficiently long time, while the impact of the shock to the time series itself would be greater than to a pure random walk.

Therefore a shock persistence is introduced as a measure for this long-run or permanent impact of shocks on the time series. This paper is organized as follows: Section 2 presents measures for persistence in univariate models. Section 3 is devoted to an empirical application of measures for persistence on the selected capital markets. Section 4 is concerned with a measurement of dependence patterns by using measures for persistence. Section 5 is again devoted to empirical applications of the shock propagation among the selected capital markets. 


\section{Persistence in Univariate Setting}

A persistence of the time series can be illustrated by the infinite polynomial of the Wold's moving average (MA) representation of the time series, $A(L)$, being evaluated at $L=1$, that is

$$
\Delta y_{t}=A(L) \varepsilon_{t}
$$

where

$$
A(L)=1+A_{1} L+A_{2} L^{2}+\ldots
$$

is a polynomial in the lag operator $L$, and $\varepsilon_{t} \sim\left(0, \sigma_{\varepsilon}^{2}\right)$ are zero mean independent residuals. An impact of the shock in period $t$ on the change level of the time series or the first difference of the time series in period $t+k$ is $A_{k}$ The impact of the shock on the level of the time series in period $t+k$ is therefore $1+\mathrm{A}_{1}+\ldots+A_{k}$. An accumulated impact of the shock on the level of the time series is the infinite sum of these MA coefficients $A$ (1). The value of $A$ (1) can then be used as a parametric approach to a measure of the persistence. For a pure random walk, we have $A(1)=$ 1 . This one follows from equations (1) and (11):

$$
A(L)=A(1)=1 \text {. }
$$

For any stationary time series, we have $A(1)=0$. It follows immediately from the following operations:

$$
\Delta y_{t}=\frac{1-L}{1-\theta L} \varepsilon_{t}, A(L)=\frac{1-L}{1-\theta L}, A(1)=0 .
$$

For time series which are neither stationary nor a pure random walk, $A(1)$ can take any value greater than zero and different from 1 . If $0<A(1)<1$, the time series would display a mean-reversion tendency. If $A(1)>1$, an unanticipated increase would be reinforced by other positive changes in the future, and the time series would continue to diverge from its pre-shock expected level. Since it is very complicated to estimate effectively the value of $A(1)$, to estimate effectively a level of the persistence via $A$ (1) is very complicated also. This is one of the reasons for using of a non-parametric approach to estimating a level of the persistence.

A non-parametric approach to estimating of a measure of the persistence is proposed by Cochrane (1988). Cochrane's measure of the persistence is a ratio of the $k$ period variance to the one period variance, being divided by $k$. The variance ratio method is non-parametric and the estimate is consequently more stable than the Wold's MA representation, $A(L)$. Cochrane's measure of a level of the persistence is known as $V_{k}$ in the following formula:

$$
\begin{aligned}
V_{k} & =\frac{1 \operatorname{Var}\left(\Delta_{k} y_{t}\right)}{k \operatorname{Var}\left(\Delta y_{t}\right)}=1+\frac{2}{\sigma_{\Delta y_{t}}^{2}} \sum_{\tau=1}^{k}\left(1-\frac{\tau}{k}\right) \operatorname{Cov}\left(\Delta y_{t}, \Delta y_{t-\tau}\right) \\
& =1+\frac{2}{\sigma_{\Delta y_{t}}^{2}} \sum_{\tau=1}^{k}\left(1-\frac{\tau}{k}\right) R_{\tau}=1+2 \sum_{\tau=1}^{k}\left(1-\frac{\tau}{k}\right) \rho_{\tau}
\end{aligned}
$$


where $\Delta_{k}$ is the $k$ period difference operator and $\Delta_{k} y_{t}=y_{t}-y_{t-k}, R_{\tau}=\operatorname{Cov}\left(\Delta y_{t}, \Delta y_{t-t}\right)$ is the $\tau$ th autocovariance in $\Delta y_{t}$ and $\rho_{\tau}=\operatorname{Cov}\left(\Delta y_{t}, \Delta y_{t-\tau}\right) / \sigma_{\Delta y_{t}}^{2}$ is the $\tau$ th autocorrelation in $\Delta y_{t}$. From (15) we can write the spectral density function as follows:

$$
2 \pi f(\omega)=1+2 \sum_{\tau=1}^{k} \rho_{\tau} \cdot \cos \left(\frac{2 \pi \tau \omega}{k+1}\right)
$$

The relationship between $V_{k}$ and $A$ (1) is as follows

$$
V_{k}=\frac{1 \operatorname{Var}\left(\Delta_{k} y_{t}\right)}{k \operatorname{Var}\left(\Delta y_{t}\right)}=\frac{1}{k} \frac{k A^{2}(1) \sigma_{\varepsilon}^{2}}{\sigma_{\Delta y_{t}}^{2}}
$$

So we can define a level of the persistence consistently in the following form:

$$
P=\lim _{k \rightarrow \infty} V_{k}=A^{2}(1) \frac{\sigma_{\varepsilon}^{2}}{\sigma_{\Delta y_{t}}^{2}} .
$$

From equations (15) and (18) we can obtain the other expression for a level of the persistence

$$
\lim _{k \rightarrow \infty} V_{k}=\lim _{k \rightarrow \infty}\left(1+2 \sum_{\tau=1}^{k}\left(1-\frac{\tau}{k}\right) \rho_{\tau}\right)=1+2 \sum_{\tau=1}^{\infty} \rho_{\tau}
$$

We assume that $\sum_{\tau=1}^{\infty}\left|\rho_{\tau}\right|<\infty$. Then it is possible to express (19) by using (16) as follows

$$
1+2 \sum_{\tau=1}^{\infty} \rho_{\tau}=2 \pi f(0)
$$

where $f($.) is a normalized spectral density function on the interval $[-\pi, \pi]$. If $P=+\infty$ then the time series is a random walk. If $P \geq 1$ then the time series is a low frequency process. If $P<1$ then the time series is a high frequency process. What is an interpretation for capital markets? If $P=+\infty$ then the capital market is represented via a random walk. It means that a shock is an inherent part of capital market returns. The capital market is then a shock persistent. If $P \geq 1$ then the capital market is represented via a low frequency process. It means that a shock is an inherent part of low frequencies in capital market returns. In other words, if a shock coming in capital market is associated with low frequencies (i.e., a trend pattern, or a capacity potency power) then it is proceeded in a very difficult and slow way. If $P<1$ then the capital market is represented via a high frequency process. If a shock coming in capital market is associated with high frequencies (i.e., an innovation pattern, or a capacity potency indication only) then stock prices are adjusted promptly. This way allows to identify both a level of the shock persistence and a level of the capital market price efficiency.

\section{Empirical Analysis of Persistence on the Capital Markets}

The time series data are composed of daily log-returns on the following indices in Table 1. The sample spans a period from January 5, 2000 till September 15, 2005. 
The data were obtained from Bloomberg data services. We estimate a level of the persistence on the selected capital markets. We consider indices of the following capital markets that are introduced in Table 1.

Table 1

\section{Selected Capital Markets}

\begin{tabular}{|r|l|l|}
\hline 1 & $P X 50$ & Prague Stock Exchange Index \\
2 & WIG & Warsaw Stock Exchange Index \\
3 & BUX & Budapest Stock Index \\
4 & NKY & Nikkei Stock Exchange Index \\
5 & $C C M P$ & Canadian Public Council Index \\
6 & $A T X$ & Australian Trade Index \\
7 & $D A X$ & German Stock Exchange Index \\
8 & UKX & London Stock Market Index \\
9 & $H S I$ & Hang Sang Index \\
10 & $A E X$ & Euronext Amsterdam Index \\
11 & $S P X$ & Standard \& Poor's 500 Index \\
12 & $C A C$ & Compagnie des Agents de Change 40 Index \\
\hline
\end{tabular}

Levels of the persistence for the selected capital markets have been computed by Cochran's approach represented by equation (15). Results are presented in the following figures in Figure 1.

Figure 1

\section{Persistence for Selected Capital Markets}

The Persistence for PX50

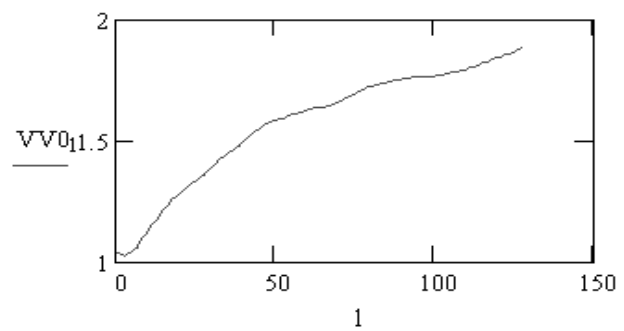

The Persistence for BUX

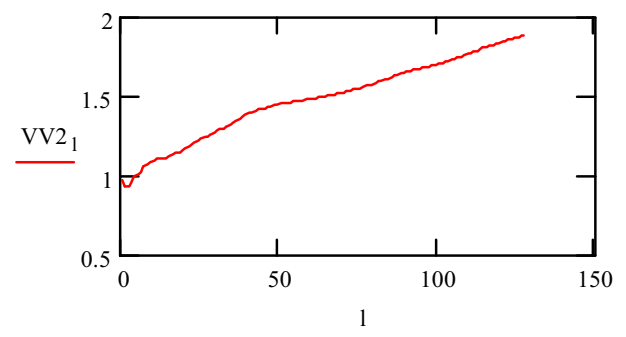

The Persistence for WIG

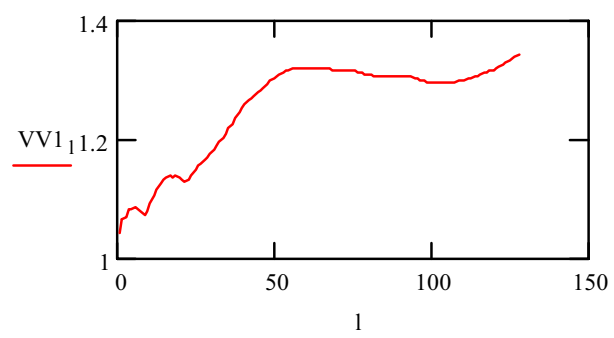

The Persistence for NKY

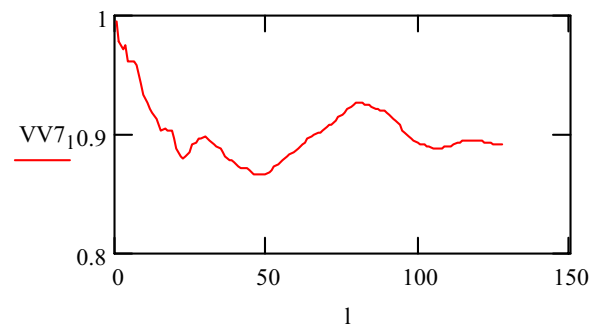



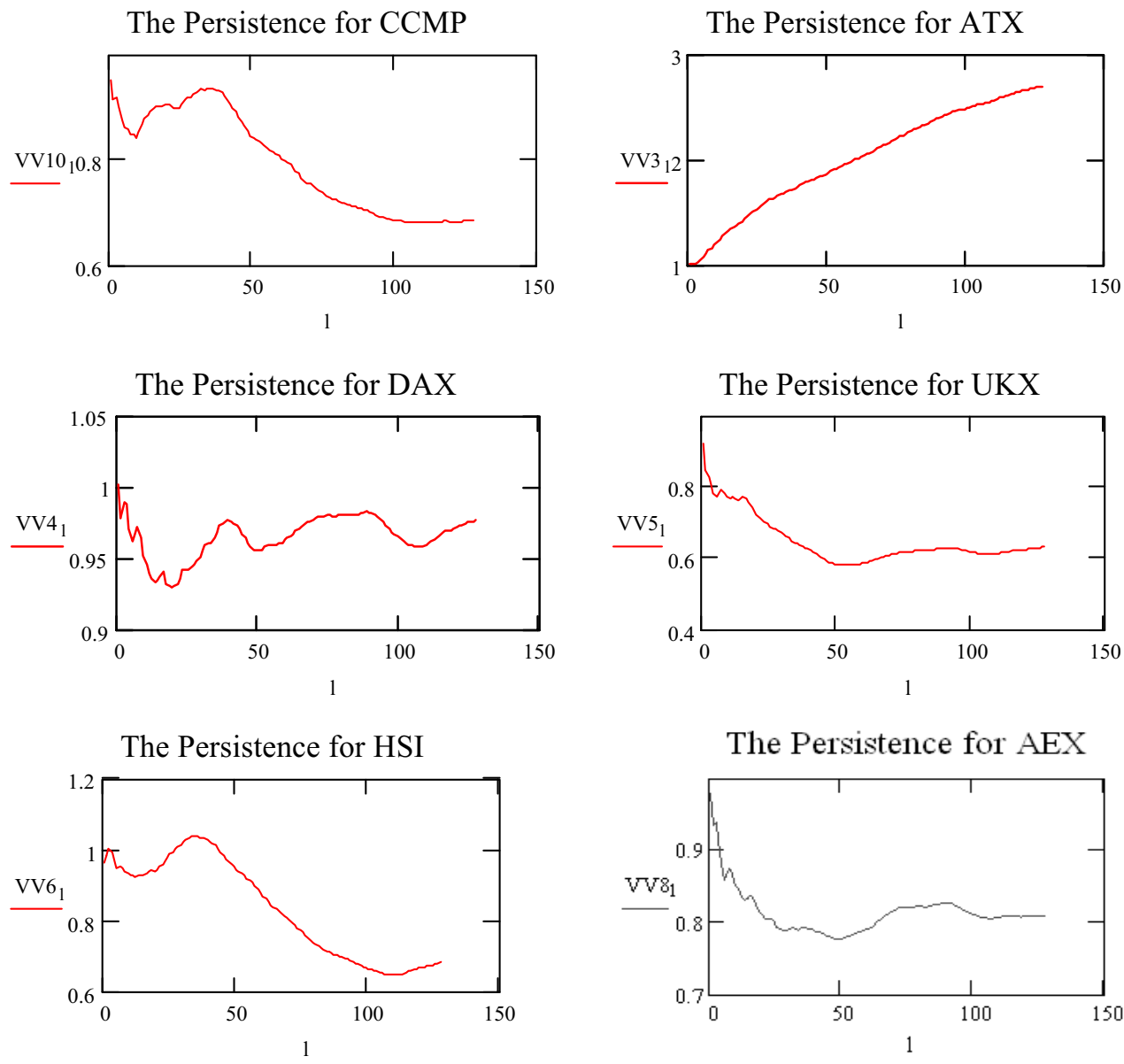

The Persistence for SPX
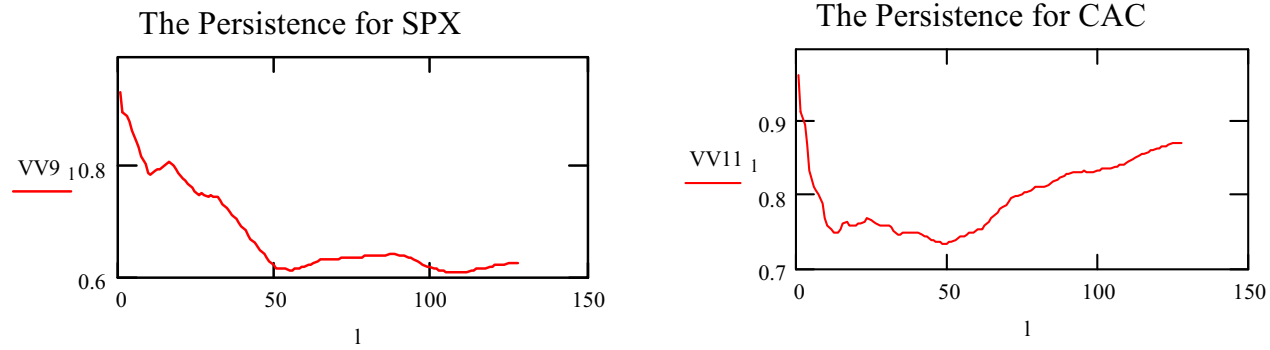

Levels of the persistence by definition (18) for the selected capital markets are introduced in Table 2. 
Table 2

Levels of the Persistence for Selected Capital Markets

\begin{tabular}{|r|r|r|}
\hline 1 & PX50 & 2.68 \\
2 & WIG & 1.73 \\
3 & BUX & 2.63 \\
4 & NKY & 0.85 \\
5 & $C C M P$ & 0.8 \\
6 & $A T X$ & 3.79 \\
7 & DAX & 0.95 \\
8 & UKX & 0.64 \\
9 & HIS & 1.07 \\
10 & AEX & 0.69 \\
11 & SPX & 0.65 \\
12 & $C A C$ & 0.91 \\
\hline
\end{tabular}

From the presented figures and Table 2 it is possible to see that the capital markets ATX, PX50, BUX, and WIG have a high level of persistence in the capital market price structures. It means that a price mechanism, for holding of efficiency in a capital market (EMH), is corrupted in these capital markets. Contrariwise, capital markets as UKX, SPX, and AEX demonstrate very efficient price mechanism for holding the capital market efficiency (EMH).

\section{Dependence Patterns among the Capital Markets}

In multivariate setting, a level of the persistence $V_{k}$, can be applied to both an identification of dependence shock patterns among capital markets and evaluation of the cross capital markets shock effects. We first adopt the infinite polynomial of the Wold's MA representation to demonstrate measures of the shock persistence, in a multivariate way similar to equation (11) as follows

$$
\Delta \mathbf{y}_{t}=\mathbf{A}(\mathrm{L}) \boldsymbol{\varepsilon}_{\mathrm{t}}, \boldsymbol{\varepsilon}_{t} \sim\left(0, \boldsymbol{\Sigma}_{\varepsilon}\right),
$$

where we use characters in bold for matrices and vectors. The expression

$$
A(L)=A_{0}+A_{1} L+A_{2} L^{2}+\ldots
$$

is an $n \times 1$ dimension vector of infinite polynomials $\Delta \boldsymbol{y}_{t}$ is an $n \times 1$ dimension vector of variables, $\varepsilon_{t}$ is an $n \times 1$ dimension vector of residuals, and $\Sigma_{\varepsilon}$ is an $n \times n$ covariance matrix of residuals. We have the multivariate measure of persistence as follows:

$$
\boldsymbol{P}=\mathbf{A}(1) \boldsymbol{\Sigma}_{\varepsilon} \boldsymbol{\Sigma}_{\Delta \mathbf{y}_{t}}^{-1} \mathbf{A}(1)^{T}
$$

which reduces to $A^{2}(1)\left(\sigma_{\varepsilon}^{2} / \sigma_{\Delta y_{t}}^{2}\right)$ in a univariate time series. To obtain multivariate measures of the shock persistence, previous studies have attempted to scale the covariance matrix of residuals by the different ways. Pesaran et al. (1993) use the conditional variance of $\Delta y_{j, t}$ (the $j$-th diagonal element of $\Sigma_{\varepsilon}$ ) to normalize the $j$-th column of the covariance matrix of residuals. Van de Gucht et al. (1996) use the unconditional variance of $\Delta y_{j, t}$ (the $j$-th diagonal element of $\Sigma_{\Delta y}$ ) to scale the $j$-th column of the covariance matrix of residuals, arguing that it is consistent with the 
univariate measure of persistence proposed by Cochrane (1988). The diagonal elements in the normalized covariance matrix are considered as a representation of the total persistence in individual capital markets. The off-diagonal elements are considered as the cross-effects of the shock persistence distributed between two capital markets. An element in the $i$-th column is the effect on the $i$-th capital market due to the shock in the $j$-th capital market. With this approach, the effect of the shock persistence on capital market $i$ due to shocks in capital market $j$ is represented by the $(i, j)$-th element in $\boldsymbol{P}$, that is $\boldsymbol{P}(i, j)$ measures the $(i, j)$-cross persistence, $\boldsymbol{P}(j, i)$ measures the $(j, i)$-cross persistence, while $\boldsymbol{P}(i, i)$ measures the total persistence of the $i$-th capital market.

As a generalizing of the non-parametric measure of the shock persistence into the multivariate setting, we define $\boldsymbol{V}_{k}$ as the $k$ period covariance matrix times the inverse of the one period covariance matrix, divided by $k$. The non-parametric measure of the shock persistence has the following form

$$
V_{k}=\frac{1}{k} \Sigma_{\Delta_{k} y_{t}} \Sigma_{\Delta y_{t}}^{-1} .
$$

Using a procedure similar to equation (15), we have:

$$
V_{k}=\left[\begin{array}{ccc}
1+2 \sum_{\tau=0}^{k-1}\left(1-\frac{\tau}{k}\right) R_{11, \tau} & \cdots & 1+2 \sum_{\tau=0}^{k-1}\left(1-\frac{\tau}{k}\right) R_{1 n, \tau} \\
\vdots & & \vdots \\
1+2 \sum_{\tau=0}^{k-1}\left(1-\frac{\tau}{k}\right) R_{n 1, \tau} & \cdots & 1+2 \sum_{\tau=0}^{k-1}\left(1-\frac{\tau}{k}\right) R_{n n, \tau}
\end{array}\right] \times\left[\begin{array}{ccc}
R_{11,0} & \cdots & R_{1 n, 0} \\
\vdots & \vdots & \vdots \\
R_{n 1,0} & \cdots & R_{n n, 0}
\end{array}\right]^{-1}
$$

where $R_{i j, \tau}=\operatorname{Cov}\left(\Delta y_{i, t}, \Delta y_{j, t-\tau}\right)$ is the covariance between $\Delta y_{i, t}$ and $\Delta y_{j, t-\tau}$ at lag $\tau$, and $R_{i j, 0}=\operatorname{Cov}\left(\Delta y_{i, t}, \Delta y_{j, t}\right)$ is the contemporaneous covariance. The elements in the first matrix on the RHS of (25) are bivariate, but the elements in $\boldsymbol{V}_{\boldsymbol{k}}$ are truly multivariate due to the second matrix on the RHS of (25). A level of the shock persistence in multivariate setting is as follows

$$
P=\lim _{k \rightarrow \infty} V_{k}=\left[\begin{array}{ccc}
1+2 \sum_{\tau=0}^{\infty} R_{11, \tau} & \cdots & 1+2 \sum_{\tau=0}^{\infty} R_{1 n, \tau} \\
\vdots & & \vdots \\
1+2 \sum_{\tau=0}^{\infty} R_{n 1, \tau} & \cdots & 1+2 \sum_{\tau=0}^{\infty} R_{n n, \tau}
\end{array}\right] \times\left[\begin{array}{ccc}
R_{11,0} & \cdots & R_{1 n, 0} \\
\vdots & \vdots & \vdots \\
R_{n 1,0} & \cdots & R_{n n, 0}
\end{array}\right]^{-1}
$$

By expression (20) we can rewrite (26) in the following expression using the bivariate spectral densities:

$$
\mathbf{P}=\lim _{k \rightarrow \infty} \mathbf{V}_{k}=2 \pi\left[\begin{array}{ccc}
h_{11}(0) & \cdots & h_{1 n}(0) \\
\vdots & & \vdots \\
h_{n 1}(0) & \cdots & h_{n n}(0)
\end{array}\right] \times\left[\begin{array}{ccc}
R_{11,0} & \cdots & R_{1 n, 0} \\
\vdots & \vdots & \vdots \\
R_{n 1,0} & \cdots & R_{n n, 0}
\end{array}\right]^{-1}
$$

where $h_{i j}$ (.) $(i, j=1, \ldots, n)$ is a cross spectrum and takes the form of 


$$
2 \pi h_{i j}(0)=1+\sum_{\tau=-\infty}^{\infty} \frac{\operatorname{Cov}\left(\Delta y_{i, t}, \Delta y_{j, t-\tau}\right)}{\sigma_{\Delta y_{i, t}} \sigma_{\Delta y_{i, t-\tau}}} .
$$

\section{Empirical Analysis of Dependence Patterns on the Capital Markets}

A level of the shock persistence, (26), takes into account an influences from all sources in the world capital markets. This measure is used for estimating the cross dependence patterns among different capital markets. Such situation, in multivariate setting, is demonstrated in the following Table 3 and Figure 2. On the $x$-axis, and the $y$-axis are labels of the capital markets 1 to 12 (see Table 1 ) that are located individually. The height of the bar on the point $(i, j)$ demonstrates a level of the shock persistence on the $i$-th capital market and due to the level of the shock persistence in the $j$-th capital market. The multivariate shock persistence analysis is more complex than the univariate shock persistence analysis. The multivariate shock persistence analysis identifies both a power of the shock and a direction of the shock propagation of the shock occurrences in the $i$-th capital market to all the others capital markets. Therefore, the multivariate shock persistence analysis is able to analyze both the sources of shocks and the shock absorbers on capital markets. Results of the empirical analysis are presented in Table 3. Dependence structures among the world capital markets are introduced in Figure 3. Moreover in Figure 3, we can investigate the dependence structures under different levels of lags $k$. The element $(i$, $i$ )-th, under $k=1$, measures a level of the shock persistence for the $(i, i)$-th capital market. The element $(i, j)$-th measures a dependence structure for the $(i, j)$ capital markets under different levels of lags $k$.

Table 3

The Level of the Persistence in Multivariate Setting for Selected Capital Markets

\begin{tabular}{|l|r|r|r|r|r|r|r|r|r|r|r|r|}
\hline & PX50 & WIG & BUX & NKY & CCMP & ATX & DAX & UKX & HSI & AEX & SPX & CAC \\
\hline PX50 & 1,419 & $-0,046$ & $-0,044$ & 0,111 & 0,063 & 0,187 & $-0,594$ & 0,026 & 0,045 & 0,616 & $-1,580$ & 0,832 \\
WIG & $-4,118$ & 0,451 & 0,224 & 0,565 & $-3,853$ & 2,919 & $-2,829$ & $-3,739$ & 1,372 & 0,027 & 8,473 & 2,247 \\
BUX & $-0,818$ & $-0,038$ & 0,402 & 0,271 & $-0,239$ & 1,370 & $-1,508$ & $-1,145$ & 0,377 & 5,941 & $-4,413$ & 0,954 \\
NKY & $-0,246$ & 0,259 & 0,081 & 0,104 & $-1,486$ & 0,946 & $-2,470$ & $-1,588$ & 0,557 & $-1,856$ & 4,620 & 2,348 \\
CCMP & 3,328 & $-0,041$ & $-0,167$ & 0,159 & $-0,383$ & $-0,102$ & $-0,607$ & $-0,150$ & 0,209 & $-2,098$ & $-0,117$ & 0,990 \\
ATX & 0,442 & $-0,028$ & 0,001 & 0,077 & 0,022 & 0,173 & $-0,439$ & $-0,028$ & 0,025 & 2,429 & $-2,229$ & 0,608 \\
DAX & $-1,425$ & 0,013 & 0,092 & 0,131 & $-0,984$ & 0,737 & $-0,070$ & $-1,049$ & 0,202 & 3,448 & $-0,279$ & 0,309 \\
UKX & 0,231 & $-0,018$ & 0,015 & 0,152 & $-0,671$ & 0,490 & $-0,077$ & $-0,320$ & 0,088 & 2,173 & $-1,251$ & 0,261 \\
HSI & $-4,045$ & 0,430 & 0,061 & 0,114 & $-3,317$ & 2,542 & $-0,686$ & $-2,966$ & 0,574 & 0,508 & 7,511 & 0,780 \\
AEX & 1,531 & $-0,041$ & $-0,059$ & 0,111 & 0,026 & 0,245 & $-0,513$ & 0,090 & 0,036 & 0,822 & $-1,962$ & 0,750 \\
SPX & 1,559 & $-0,045$ & $-0,046$ & 0,121 & $-0,140$ & 0,035 & $-0,391$ & $-0,036$ & 0,044 & 0,267 & $-1,024$ & 0,691 \\
CAC & 1,145 & 0,027 & $-0,001$ & 0,123 & $-1,172$ & $-0,166$ & 0,111 & $-0,224$ & 0,119 & 1,742 & $-0,628$ & 0,027 \\
\hline
\end{tabular}

In Figure 3, we can observe that SPX and AEX are the main parts in bearing of shocks in capital markets. In this one, we can also observe an evolution of dependence structures both short-run and long-run intervals among capital markets. 


\section{Conclusions}

The paper has been focused on a research of the dependence structures on the capital markets by measures of the persistence. In univariate systems, an empirical analysis of persistence has shown that the capital markets UKX, AEX, and SPX are characterized by a low level of the persistence under all analyzed lags. From these analyses it is possible to show a level of the capital market efficiency. In multivariate systems, an empirical analysis of persistence has shown that capital markets change dependence structures in different periods of lags. The negative tail dependence in the returns on the PX50, WIG, and UKX indices, representing the Prague Stock Exchange Index, the Warsaw Stock Exchange Index, and the London Stock Market Index was found. The positive tail dependence in the returns on the SPX, WIG, and HSI indices, representing the Standard \& Poor's 500 Index, the Warsaw Stock Exchange Index, and the Hang Sang Index was found. This analysis enables to construct more deep hedging structure of agent portfolio securities.

Figure 2

Dependence Structures Among the World Capital Markets

\section{Levels of the Persistence on the World Capital Markets}

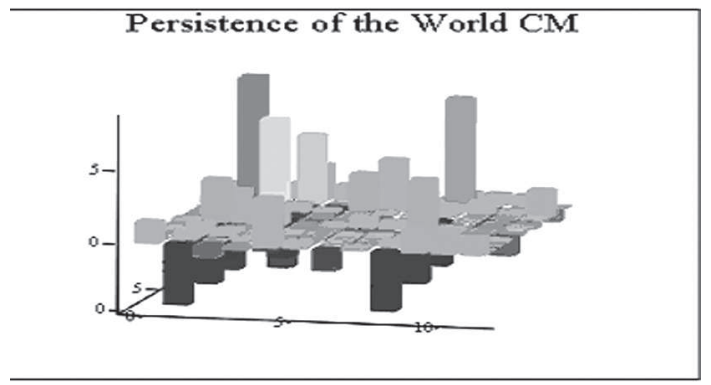

Figure 3

Dependence Structures Among the World Capital Markets for Different Lags $K=2,2^{2}, \ldots, 2^{6}$
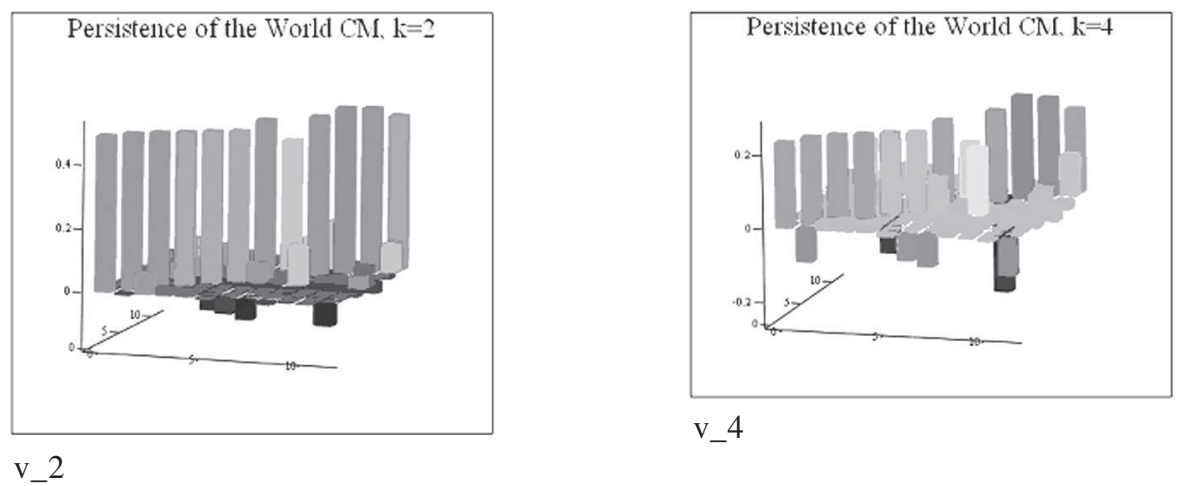


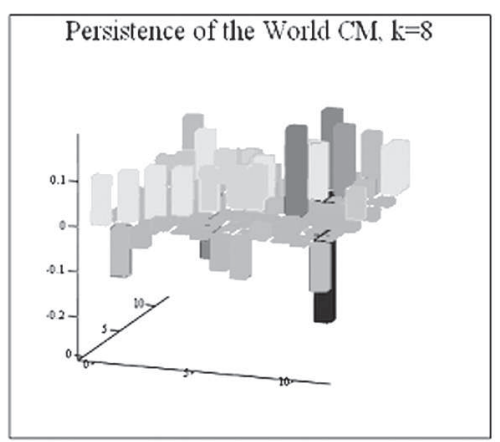

v_8

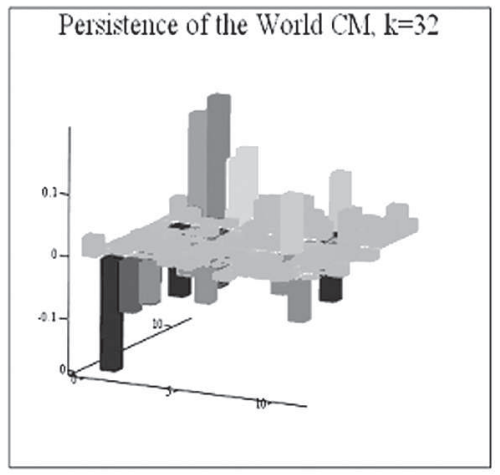

v_32

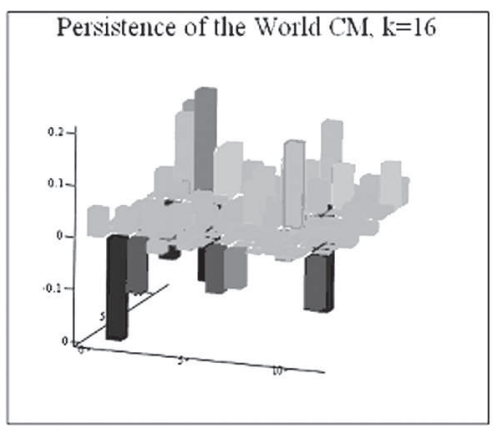

v_16

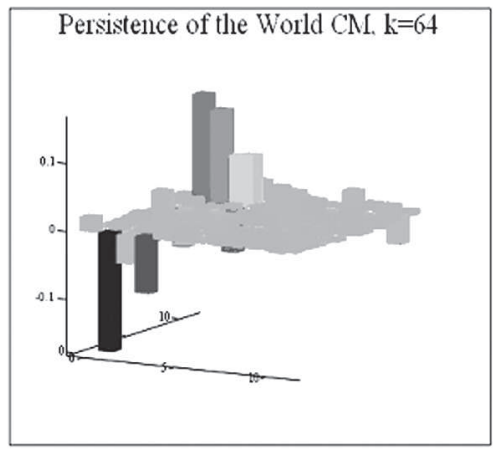

v_64

Acknowledgements: I would like to give many thanks to anonymous referee for very fruitful comments.

\section{References}

Box, G. E. P., Jenkins, G. E. M. (1976), Time Series Analysis: Forecasting and Control. San Francisco: Holden-Day.

Cochrane, J. H. (1988), How Big is the Random Walk in GDP? Journal of Political Economy, 96, pp. 893-920,

Fama, E. (1965), The Behaviour of Stock Prices. Journal of Business, 38, pp. 34-105, (1970), Efficient Capital Markets. Journal of Finance, 25, pp. 383-417.

Hu, L. (2005), Dependence Patterns across Financial Markets: a Mixed Copula Approach. Applied Financial Economics.

Mandelbrot, B. (1963), The Variation of Certain Speculative Prices. Journal of Business, 36, pp. 394-419.

Pesaran, M. H., Pierse, R. G., Lee, K. C. (1993), Persistence, Cointegration and Aggregation: A Disaggregated Analysis of Output Fluctuations in the US Economy. Journal of Econometrics, 56, pp. 67-88.

Schweitzer, B., Wolf, E. (1981), On Non-Parametric Measures of Dependence for Random Variables. Annals of Statistics, 9, pp. 879-85.

Van de Gucht, L. M., Dekimpe, M. G., Kwok, C. C. Y. (1996), Persistence of Foreign Exchange Rates. Journal of International Money and Finance, 15, pp. 191-220. 